\title{
Aortic Insufficiency in Patients With Sustained Left Ventricular Systolic Dysfunction After Axial Flow Assist Device Implantation
}

Teruhiko Imamura, MD, PhD; Koichiro Kinugawa, MD, PhD; Takeo Fujino, MD, PhD; Toshiro Inaba, MD, PhD; Hisataka Maki, MD, PhD; Masaru Hatano, MD; Osamu Kinoshita, MD, PhD; Kan Nawata, MD, PhD; Shunei Kyo, MD, PhD; Minoru Ono, MD, PhD

Background: Predicting the occurrence of aortic insufficiency (AI) during left ventricular assist device (LVAD) support has remained unsolved.

Methods and Results: We enrolled 52 patients who had received continuous flow LVAD (14 axial and 38 centrifugal pumps) and who been followed for $\geq 6$ months between Jun 2006 and Dec 2013. Native aortic valve (AV) opening was observed in 18 patients (35\%) with improved LV systolic function, and none of them had Al. On multivariate logistic regression analysis preoperative shorter heart failure duration was the only independent predictor of postoperative native $A V$ opening ( $P=0.042$; odds ratio [OR], 0.999). Of the remaining 34 patients (65\%) with closed $A V, 11$ had $\mathrm{Al}$ with enlargement of the aortic root and narrow pulse pressure. Among those with closed $\mathrm{AV}$, axial pump use $(n=13)$ was the only significant predictor of the development of $A l(P=0.042$; OR, 4.950). Patients with Al had lower exercise capacity and a higher readmission rate than those without AI during 2 -year LVAD support (55\% vs. $8 \%$; $\mathrm{P}<0.001)$.

Conclusions: Native AV opening during LVAD support is profoundly associated with reversal of LV systolic function, especially in patients with preoperative shorter heart failure duration. Among those in whom the native AV remains closed, low pulsatility of axial flow pump may facilitate aortic root remodeling and post-LVAD AI development that results in worse clinical outcome. (Circ $J$ 2015; 79: 104-111)

Key Words: Centrifugal; EVAHEART; HeartMate II; Ventricular assist device

A lthough the outcome of left ventricular assist device (LVAD) treatment has been improving thanks to the development of the continuous flow (CF) pump, patient selection, and perioperative management, ${ }^{1-5}$ aortic insufficiency (AI) remains an unsolved problem during LVAD support. ${ }^{6} \mathrm{AI}$ leads to reduced forward cardiac output and end-organ hypoperfusion, ${ }^{7}$ which eventually results in poor outcome. ${ }^{8,9}$

\section{Editorial $\mathrm{p} \mathbf{4 3}$}

There have been no established treatments for AI thus far. Although some authors recently reported successful replacement or plasty of aortic valve (AV) for progressed AI, such procedures are invasive and still have various fatal complications. ${ }^{10}$ Preoperative risk stratification and successful prevention of $\mathrm{AI}$ is an inevitable concern for successful long-term LVAD treatment.
AI is a multifactorial phenomenon, but continuous closure of native AV would be a key for the development of AI. ${ }^{10}$ Although several studies proposed higher age, usage of $\mathrm{CF}$ pump, or preoperative lower left ventricular ejection fraction (LVEF) as risk factors for AI, ${ }^{9,11-13}$ the precise mechanism has remained unknown. Therefore, the aim of the present study was to identify the perioperative factors affecting AI during $\mathrm{CF}$ LVAD support.

\section{Patients}

We retrospectively enrolled 52 patients with stage D heart failure (HF) who had received CF LVAD (14 axial pumps: HeartMate II, $\mathrm{n}=11$; Jarvik 2000, $\mathrm{n}=3 ; 38$ centrifugal pumps: EVAHEART, $n=24$; DuraHeart, $n=14$ ) as a bridge to heart transplantation and who had been followed at the University

Received August 24, 2014; revised manuscript received October 1, 2014; accepted October 5, 2014; released online November 7, 2014 Time for primary review: 29 days

Department of Therapeutic Strategy for Heart Failure (T. Imamura, K.K., S.K.), Department of Cardiovascular Medicine (T.F., T. Inaba, H.M., M.H.), Department of Thoracic Surgery (O.K., K.N., M.O.), Graduate School of Medicine, University of Tokyo, Tokyo, Japan

Mailing address: Koichiro Kinugawa, MD, PhD, Department of Therapeutic Strategy for Heart Failure, Graduate School of Medicine, University of Tokyo, 7-3-1 Hongo, Bunkyo-ku, Tokyo 113-8655, Japan. E-mail: kinugawa-tky@umin.ac.jp

ISSN-1346-9843 doi:10.1253/circj.CJ-14-0944

All rights are reserved to the Japanese Circulation Society. For permissions, please e-mail: cj@j-circ.or.jp 
of Tokyo Hospital for at least 6 months between 2006 and 2013. Those with concomitant AV replacement were excluded. No patients had received concomitant right VAD implantation.

All patients were treated preoperatively with guideline-directed medical therapy consisting of $\beta$-blocker, angiotensin-converting enzyme inhibitor, and aldosterone antagonist unless contraindicated and doses of these drugs were titrated considering patient hemodynamics.

The rotation speed of LVAD was optimized as low as possible considering patient hemodynamics and interventricular septum shift observed in regular echocardiography. Written informed consent was obtained before LVAD implantation from all patients. The study protocol was approved by the Ethics Committee of the Graduate School of Medicine, University of Tokyo [application number 779 (1)].

\section{Preoperative Variables}

Preoperative baseline data including patient demographics and laboratory parameters were obtained within $24 \mathrm{~h}$ before surgery. Hemodynamic and echocardiographic parameters were obtained within 1 week before operation or before initiation of intraaortic balloon pump. LVEF was calculated using the biplane Simpson method. Valvular regurgitation was classified into 5 grades: 0 , none; 1 , trace; 2 , mild; 3 , moderate; 4, severe. Valsalva sinus and aortic root diameters were measured in all patients on long axis view. HF duration was defined as the time between HF diagnosis and LVAD implantation.

\section{Postoperative Variables}

Hemodynamic examination was carried out in all patients at 5 weeks after operation. VAD flow was estimated using algorithms of each device. Transthoracic echocardiography was performed regularly, and AI was defined as aortic regurgitation $\geq$ grade 2 at 6 months. To determine the frequency of the native $\mathrm{AV}$ opening, we counted the number of native AV openings per native heart rate over at least $1 \mathrm{~min}$. We defined native AV opening for $<30 \%$ of the native heart rate as "remaining closed". Computed tomography was done in all patients, and the height from native AV to the VAD outflow graft anastomosed at the ascending aorta was measured. Peak oxygen consumption during cardiopulmonary exercise test and 6-min walk distance were measured at 6 months after operation. Readmission due to cardiovascular events was counted during 2 years after the first discharge.

\section{Statistical Analysis}

All statistical analysis was done using PASW Statistics 18 (SPSS, Chicago, IL, USA). All hypothesis tests were 2-tailed, and used $\mathrm{P}<0.05$ as significant. All data are expressed as mean \pm SD unless otherwise specified. Continuous variables were compared using unpaired t-test or Mann-Whitney U-test as appropriate. Categorical variables were compared using chi-squared test or Fisher's exact test as appropriate. Logistic regression analysis was used to calculate significant predictors for AI or native AV opening. Kaplan-Meier analysis was performed to compare readmission-free rate among those with/ without AI. Variables significant on univariate analysis at $\mathrm{P}<0.05$ were used in multivariate analysis.

\section{Results}

\section{Al and Native AV Opening During LVAD Support}

All 18 patients $(35 \%)$ who had achieved native AV opening, did not have AI (group X; Table 1). Among those with continuous AV closure, 23 patients (44\%) had no AI (group Y), whereas

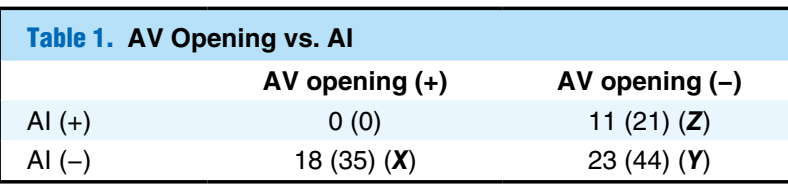

Data given as $n(\%) .{ }^{*} P<0.02$ (chi-squared test). Al, aortic insufficiency; AV, aortic valve; $\mathrm{X}$, native AV opening; $\mathrm{Y}$, no Al during continuous AV closure; $\mathrm{Z}$, Al during continuous AV closure.

$11(21 \%)$ did have AI (group Z).

\section{Preoperative Predictors for Postoperative Native AV Opening}

The mean age was $41 \pm 13$ years, and 43 patients (83\%) were male (Table 2). All patients were dependent on continuous inotrope infusion and received elective LVAD implantation. No patients had AI preoperatively. On multivariate logistic regression preoperative shorter $\mathrm{HF}$ duration was the only significant predictor for native AV opening (Table 2).

\section{Postoperative Characterization of Native AV Opening}

Compared with those whose native AV remained closed, wider pulse pressure and higher systolic blood pressure were observed in patients with native AV opening (Table 3). Improved LVEF with smaller Valsalva sinus or aortic root was also associated with native AV opening (Table 3). Postoperative medical treatment was similar among all patients.

\section{Predictors for Development of Al in Continuous AV Closure} In patients with continuous AV closure $(n=34)$, univariate logistic regression analyses indicated that use of axial pump was the only significant predictor for $\mathrm{AI}(\mathrm{P}=0.042$; odds ratio, 4.950; Table 4). There were no significant statistical differences in patient background except for gender and Valsalva sinus diameter between the axial and centrifugal pumps (Table S1). Notably, larger Valsalva sinus diameter was observed in patients with centrifugal pump.

\section{Postoperative Characterization of Al}

Among postoperative variables, narrow pulse pressure along with enlargement of Valsalva sinus and aortic root were associated with the development of AI during LVAD support $(\mathrm{P}<0.05$ in all; Table 5).

Patients with centrifugal LVAD had significantly wider pulse pressure than those with axial LVAD (Table S2). There were no significant differences in estimated VAD flow between axial and centrifugal pumps. Patients with axial pump experienced more enlargement in Valsalva sinus and aortic root during LVAD support (Table S2).

\section{Clinical Course vs. Presence of Al}

Patients with AI had lower peak oxygen consumption during cardiopulmonary exercise test compared to those without AI (Figure A; $11.0 \pm 3.3$ vs. $14.4 \pm 3.5 \mathrm{ml} \cdot \mathrm{min}^{-1} \cdot \mathrm{kg}^{-1}, \mathrm{P}=0.004$ ) and shorter 6-min walk distance (Figure B; $328 \pm 84$ vs. $407 \pm 66 \mathrm{~m}$, $\mathrm{P}=0.001)$ at 6 months after LVAD implantation. Patients with $\mathrm{AI}$ had a higher readmission rate due to cardiovascular events than those without AI during the 2-year LVAD support period (Figure C; $55 \%$ vs. 8\%, $\mathrm{P}<0.001$ ). There was no difference in 2-year survival under LVAD support regardless of AI (Figure D; $\mathrm{P}=0.856)$. The prevalence of $\mathrm{AI}$ was $6 \%$ at 1 month $(3 / 52)$, $13 \%$ at 3 months (7/52), $21 \%$ at 6 months (11/52), and $18 \%$ at 1 year (7/40). No significant AI newly developed after the first 6 months. LVAD was explanted in 2 patients (4\%), and 7 pa- 
Table 2. Preoperative Predictors for Postoperative Native AV Opening

\begin{tabular}{|c|c|c|c|c|c|c|c|c|c|}
\hline \multirow{2}{*}{$\begin{array}{l}\text { Preoperative } \\
\text { parameters }\end{array}$} & \multirow{2}{*}{$\begin{array}{l}\text { Total } \\
(n=52)\end{array}$} & \multirow{2}{*}{$\begin{array}{c}\begin{array}{c}\text { AV opening } \\
(+)(n=18)\end{array} \\
\text { Group X }\end{array}$} & \multirow{2}{*}{$\begin{array}{l}\text { AV opening } \\
(-)(n=34) \\
\text { Group } Y+Z\end{array}$} & \multicolumn{3}{|c|}{ Univariate analysis } & \multicolumn{3}{|c|}{ Multivariate analysis } \\
\hline & & & & P-value & OR & $95 \% \mathrm{Cl}$ & P-value & OR & $95 \% \mathrm{Cl}$ \\
\hline \multicolumn{10}{|l|}{$\begin{array}{l}\text { Demographic } \\
\text { parameters }\end{array}$} \\
\hline Age (years) & $41 \pm 13$ & $40 \pm 11$ & $42 \pm 13$ & 0.584 & 0.987 & $0.941-1.035$ & & & \\
\hline Male & $43(83)$ & $17(94)$ & $26(76)$ & 0.068 & 0.128 & $0.048-1.020$ & & & \\
\hline Ischemic etiology & $5(10)$ & $4(22)$ & $1(3)$ & 0.054 & 9.429 & $0.966-92.06$ & & & \\
\hline $\begin{array}{l}\text { Body surface } \\
\text { area }\left(\mathrm{m}^{2}\right)\end{array}$ & $1.7 \pm 0.2$ & $1.72 \pm 0.11$ & $1.65 \pm 0.17$ & 0.141 & 20.13 & $0.370-1094$ & & & \\
\hline HF duration (days) & $2,138 \pm 1,721$ & $1,122 \pm 1,650$ & $2,677 \pm 1,522$ & $0.024^{*}$ & 0.999 & $0.999-1.000$ & $0.042^{*}$ & 0.999 & $0.999-1.000$ \\
\hline \multicolumn{10}{|c|}{$\begin{array}{l}\text { Device selection } \\
\text { and valve plasty }\end{array}$} \\
\hline Axial pump & $14(27)$ & $1(6)$ & $13(38)$ & $0.030^{*}$ & 0.095 & $0.011-1.010$ & 0.154 & 0.098 & $0.014-1.176$ \\
\hline Centrifugal pump & $38(73)$ & $17(94)$ & $21(62)$ & - & - & - & - & - & - \\
\hline Mitral valve plasty & $20(38)$ & $7(39)$ & $13(38)$ & 0.963 & 0.973 & $0.301-3.144$ & & & \\
\hline $\begin{array}{l}\text { Tricuspid } \\
\text { annuloplasty }\end{array}$ & $22(42)$ & $10(56)$ & $12(4)$ & 0.163 & 0.436 & $0.136-1.400$ & & & \\
\hline \multicolumn{10}{|l|}{$\begin{array}{l}\text { Hemodynamic } \\
\text { parameters }\end{array}$} \\
\hline $\mathrm{SBP}(\mathrm{mmHg})$ & $85 \pm 13$ & $86 \pm 12$ & $85 \pm 14$ & 0.885 & 1.003 & $0.961-1.048$ & & & \\
\hline $\mathrm{DBP}(\mathrm{mmHg})$ & $56 \pm 12$ & $54 \pm 12$ & $57 \pm 11$ & 0.382 & 0.977 & $0.927-1.030$ & & & \\
\hline $\begin{array}{l}\text { Heart rate } \\
\text { (beats/min) }\end{array}$ & $87 \pm 15$ & $85 \pm 14$ & $89 \pm 14$ & 0.264 & 1.025 & $0.981-1.072$ & & & \\
\hline mPAP $(\mathrm{mmHg})$ & $32 \pm 8$ & $35 \pm 10$ & $31 \pm 10$ & 0.140 & 1.046 & $0.985-1.111$ & & & \\
\hline PCWP (mmHg) & $23 \pm 8$ & $26 \pm 6$ & $22 \pm 9$ & 0.181 & 1.055 & $0.975-1.142$ & & & \\
\hline $\begin{array}{l}\text { Cardiac index } \\
\left(\mathrm{L} \cdot \mathrm{min}^{-1} \cdot \mathrm{m}^{-2}\right)\end{array}$ & $2.0 \pm 0.4$ & $2.0 \pm 0.5$ & $2.0 \pm 0.4$ & 0.655 & 0.738 & $0.187-2.917$ & & & \\
\hline mRAP $(\mathrm{mmHg})$ & $10 \pm 5$ & $12 \pm 4$ & $8 \pm 5$ & $0.014^{*}$ & 1.192 & $1.037-1.372$ & 0.274 & 1.045 & $0.956-1.654$ \\
\hline RVSWI $\left(\mathrm{g} / \mathrm{m}^{2}\right)$ & $7.2 \pm 3.4$ & $6.8 \pm 3.3$ & $7.4 \pm 3.6$ & 0.538 & 0.946 & $0.795-1.127$ & & & \\
\hline \multicolumn{10}{|l|}{$\begin{array}{l}\text { Echocardiographic } \\
\text { parameters }\end{array}$} \\
\hline LVDd (mm) & $75 \pm 15$ & $75 \pm 16$ & $75 \pm 14$ & 0.984 & 1.000 & $0.962-1.040$ & & & \\
\hline LVEF (\%) & $19 \pm 8$ & $22 \pm 6$ & $19 \pm 9$ & 0.259 & 1.042 & $0.970-1.118$ & & & \\
\hline AR (grade) & $0.4 \pm 0.6$ & $0.2 \pm 0.4$ & $0.5 \pm 0.7$ & 0.058 & 0.269 & $0.072-1.010$ & & & \\
\hline MR (grade) & $2.3 \pm 1.0$ & $2.2 \pm 1.0$ & $2.4 \pm 1.1$ & 0.669 & 0.888 & $0.515-1.532$ & & & \\
\hline TR (grade) & $1.5 \pm 0.7$ & $1.6 \pm 0.6$ & $1.5 \pm 0.8$ & 0.686 & 1.177 & $0.534-2.592$ & & & \\
\hline $\begin{array}{l}\text { Valsalva sinus } \\
\text { diameter }(\mathrm{mm})\end{array}$ & $29 \pm 3$ & $30 \pm 4$ & $29 \pm 3$ & 0.136 & 1.145 & $0.958-1.368$ & & & \\
\hline $\begin{array}{l}\text { AV ring diameter } \\
(\mathrm{mm})\end{array}$ & $20 \pm 2$ & $20 \pm 1$ & $19 \pm 2$ & 0.088 & 1.365 & $0.955-1.951$ & & & \\
\hline \multicolumn{10}{|l|}{$\begin{array}{l}\text { Laboratory } \\
\text { parameters }\end{array}$} \\
\hline Hemoglobin (g/dl) & $11.5 \pm 2.0$ & $11.4 \pm 1.5$ & $11.6 \pm 2.3$ & 0.682 & 0.940 & $0.699-1.264$ & & & \\
\hline Platelets $\left(\times 10^{3} / \mu \mathrm{l}\right)$ & $21.1 \pm 7.1$ & $20.6 \pm 8.5$ & $20.3 \pm 6.8$ & 0.898 & 1.005 & $0.928-1.088$ & & & \\
\hline $\begin{array}{l}\text { Serum albumin } \\
(\mathrm{g} / \mathrm{dl})\end{array}$ & $3.5 \pm 0.6$ & $3.4 \pm 0.5$ & $3.6 \pm 0.7$ & 0.178 & 0.530 & $0.201-1.346$ & & & \\
\hline $\begin{array}{l}\text { Serum sodium } \\
(\mathrm{mEq} / \mathrm{L})\end{array}$ & $134 \pm 5$ & $132 \pm 7$ & $134 \pm 4$ & 0.234 & 0.937 & $0.842-1.043$ & & & \\
\hline $\begin{array}{l}\text { Serum creatinine } \\
(\mathrm{mg} / \mathrm{dl})\end{array}$ & $1.1 \pm 0.5$ & $1.0 \pm 0.3$ & $1.2 \pm 0.6$ & 0.249 & 0.451 & $0.116-1.746$ & & & \\
\hline $\begin{array}{l}\text { Serum total } \\
\text { bilirubin (mg/dl) }\end{array}$ & $1.6 \pm 1.3$ & $2.1 \pm 1.6$ & $1.4 \pm 1.0$ & 0.065 & 1.611 & $0.971-2.673$ & & & \\
\hline $\begin{array}{l}\text { Plasma BNP } \\
(\mathrm{pg} / \mathrm{ml})\end{array}$ & $877 \pm 667$ & $872 \pm 630$ & $880 \pm 695$ & 0.968 & 1.000 & $0.999-1.001$ & & & \\
\hline
\end{tabular}

Data given as mean \pm SD or $\mathrm{n}(\%) .{ }^{*} \mathrm{P}<0.05$ (logistic regression). ACEI, angiotensin-converting enzyme inhibitor; AR, aortic valve regurgitation; $\mathrm{AV}$, aortic valve; BNP, B-type natriuretic peptide; $\mathrm{Cl}$, confidence interval; DBP, diastolic blood pressure; HF, heart failure; LVDd, left ventricular (LV) diastolic diameter; LVEF, LV ejection fraction; $\mathrm{mPAP}$, mean pulmonary artery pressure; MR, mitral valve regurgitation; $\mathrm{mRAP}$, mean right atrial pressure; OR, odds ratio; PCWP, pulmonary capillary wedge pressure; RVSWI, right ventricular stroke work index; SBP, systolic blood pressure; TR, tricuspid valve regurgitation. 


\begin{tabular}{|c|c|c|c|}
\hline \multirow[t]{2}{*}{ Parameters } & $\begin{array}{c}\text { AV opening } \\
(+)(n=18)\end{array}$ & $\begin{array}{l}\text { AV opening } \\
(-)(n=34)\end{array}$ & \multirow[t]{2}{*}{ P-value } \\
\hline & Group X & Group $Y+Z$ & \\
\hline \multicolumn{4}{|l|}{ Postoperative hemodynamics } \\
\hline Heart rate (beats/min) & $84 \pm 9$ & $82 \pm 10$ & 0.587 \\
\hline $\mathrm{mPAP}(\mathrm{mmHg})$ & $18 \pm 7$ & $16 \pm 5$ & 0.364 \\
\hline PCWP (mmHg) & $9 \pm 6$ & $8 \pm 4$ & 0.695 \\
\hline Cardiac index $\left(\mathrm{L} \cdot \mathrm{min}^{-1} \cdot \mathrm{m}^{-2}\right)$ & $2.5 \pm 0.6$ & $2.6 \pm 0.6$ & 0.694 \\
\hline mRAP $(\mathrm{mmHg})$ & $9 \pm 6$ & $7 \pm 4$ & 0.458 \\
\hline RVSWI $\left(g / m^{2}\right)$ & $4.1 \pm 2.1$ & $3.9 \pm 1.9$ & 0.738 \\
\hline Pulse pressure $(\mathrm{mmHg})$ & $24 \pm 8$ & $15 \pm 7$ & $0.003^{*}$ \\
\hline $\mathrm{SBP}(\mathrm{mmHg})$ & $93 \pm 6$ & $89 \pm 6$ & $0.042^{*}$ \\
\hline $\mathrm{DBP}(\mathrm{mmHg})$ & $69 \pm 7$ & $73 \pm 9$ & 0.078 \\
\hline Estimated VAD flow (L/min) & $4.1 \pm 1.3$ & $3.5 \pm 0.8$ & 0.097 \\
\hline Height of outflow cannula $(\mathrm{cm})$ & $2.2 \pm 0.3$ & $2.3 \pm 0.3$ & 0.395 \\
\hline Carvedilol at 6 months (mg/day) & $14.2 \pm 5.5$ & $15.6 \pm 13.7$ & 0.600 \\
\hline Enalapril at 6 months (mg/day) & $2.1 \pm 2.0$ & $1.6 \pm 1.8$ & 0.323 \\
\hline \multicolumn{4}{|l|}{ Postoperative echocardiography } \\
\hline LVDd (mm) & $67 \pm 13$ & $63 \pm 16$ & 0.378 \\
\hline \%change in LVDd (\%) & $-11 \pm 12$ & $-15 \pm 17$ & 0.303 \\
\hline LVEF (\%) & $28 \pm 14$ & $17 \pm 7$ & $0.012^{*}$ \\
\hline \%change in LVEF (\%) & $34 \pm 83$ & $2 \pm 47$ & 0.139 \\
\hline MR (grade) & $0.6 \pm 0.7$ & $0.6 \pm 0.9$ & 0.984 \\
\hline TR (grade) & $0.6 \pm 0.6$ & $1.1 \pm 0.9$ & 0.067 \\
\hline Valsalva sinus diameter (mm) & $29 \pm 3$ & $31 \pm 4$ & 0.253 \\
\hline$\%$ change in Valsalva sinus diameter (\%) & $-1 \pm 3$ & $8 \pm 7$ & $0.001^{*}$ \\
\hline $\mathrm{AV}$ ring diameter $(\mathrm{mm})$ & $20 \pm 2$ & $21 \pm 2$ & 0.225 \\
\hline \%change in AV ring diameter (\%) & $-2 \pm 5$ & $6 \pm 9$ & $0.002^{*}$ \\
\hline
\end{tabular}

Data given as mean $\pm S D$. ${ }^{*} P<0.05$ (unpaired t-test or Mann-Whitney test). LVAD, LV assist device; VAD, ventricular assist device. Other abbreviations as in Table 2.

tients (13\%) underwent heart transplant during the study period.

$\mathrm{AV}$ condition and clinical course during the study period among the 4 devices (EVAHEART, DuraHeart, HeartMate II, and Jarvik 2000) are summarized in Table 6.

\section{Discussion}

All patients who achieved native AV opening were free from AI development during 6 months of CF LVAD support. On logistic regression analysis preoperative shorter HF duration was associated with AV opening accompanied by improved LVEF during LVAD support. Among those with continuous AV closure, more patients with axial LVAD had AI along with less pulsatility, and aortic root remodeling. Patients with AI had worse clinical course than those without AI.

\section{Definition of Al During LVAD Support}

We considered that AI $\geq$ grade 2 was hemodynamically significant, because AI after LVAD implantation was typically continuous throughout the cardiac cycle and the regurgitant fraction was approximately twice as much as that in the patients without VAD support. ${ }^{12}$ Patients with preoperative AI $\geq$ grade 2 received concomitant $\mathrm{AV}$ replacement at the time of LVAD implantation, and such patients were excluded from this study. As a result, all AI during LVAD support were de novo. Considering that all AI accompanied continuous AV closure in the present study (Table 1), AI was analyzed in a stepwise manner, that is, continuous AV closure at first, and then the development of AI.

We evaluated AI at 6 months after LVAD implantation, which was relatively earlier than the observation periods used in other studies. ${ }^{8} 11,12,14 \mathrm{We}$ chose 6 months for evaluation of endpoints because death or explantation of LVAD occurred in some patients soon after 6 months. Moreover, no significant AI was newly developed after the first 6 months of LVAD implantation in the present study. Although AI is a progressive phenomenon, its onset may be determined within the first 6 months after LVAD implantation with closed native AV.

\section{Prevalence of Al and Optimization of Rotation Speed}

Although the prevalence of AI varied in each report, probably because of variation in definition, timing of evaluation, device type, patient background, or perioperative management, most authors reported an AI prevalence of $20-50 \%$ within the first year. ${ }^{8,9,11,12,14}$ Jorde et al argued that optimization of rotation speed as low as possible so as to accomplish native AV opening eventually repressed development of AI. ${ }^{8}$ We here defined native AV opening at $<30 \%$ of the native heart rate as "remaining closed", because Slaughter et al noted that AV opening at least once per 3 native heart beats may be sufficient to avoid development of AI. ${ }^{15} \mathrm{We}$ carried out such optimization in all patients during scheduled hemodynamic examination and serial transthoracic echocardiography, but still observed a prevalence of AI of 33\% within 6 months. Lowering rotation speed down to the level of native AV opening sometimes limited maintenance of sufficient cardiac output. Because there has 
Table 4. Predictors for Development of Al in Continuous AV Closure

Continuous AV closure $(n=34)$

Preoperative parameters

Demographic parameters

\begin{tabular}{|c|c|c|c|c|c|}
\hline Age (years) & $42 \pm 10$ & $42 \pm 14$ & 0.933 & 1.002 & $0.947-1.062$ \\
\hline Male & $7(63)$ & $18(78)$ & 0.370 & 0.486 & $0.100-2.356$ \\
\hline Etiology of ischemia & $1(9)$ & $0(0)$ & - & - & - \\
\hline Body surface area $\left(\mathrm{m}^{2}\right)$ & $1.6 \pm 0.2$ & $1.7 \pm 0.2$ & 0.601 & 0.318 & $0.004-23.27$ \\
\hline HF duration (days) & $2,986 \pm 1,512$ & $2,530 \pm 1,537$ & 0.412 & 1.000 & $1.000-1.001$ \\
\hline \multicolumn{6}{|l|}{ Medications } \\
\hline Cumulative dose of $\beta$-blocker (g) & $31 \pm 27$ & $19 \pm 19$ & 0.184 & 1.026 & $0.984-1.060$ \\
\hline Cumulative dose of ACEI (g) & $13 \pm 23$ & $9 \pm 13$ & 0.413 & 1.018 & $0.976-1.063$ \\
\hline \multicolumn{6}{|l|}{ Device selection and valve plasty } \\
\hline Axial pump & $7(63)$ & $6(26)$ & $0.042^{*}$ & 4.950 & $1.062-23.26$ \\
\hline Centrifugal pump & $4(36)$ & $17(74)$ & - & - & - \\
\hline Mitral valve plasty & $3(27)$ & $8(35)$ & 0.417 & 0.375 & $0.035-3.999$ \\
\hline Tricuspid annuloplasty & $3(27)$ & $7(30)$ & 0.699 & 0.643 & $0.068-6.056$ \\
\hline \multicolumn{6}{|l|}{ Hemodynamic parameters } \\
\hline $\mathrm{SBP}(\mathrm{mmHg})$ & $90 \pm 9$ & $83 \pm 16$ & 0.209 & 1.035 & $0.981-1.093$ \\
\hline $\mathrm{DBP}(\mathrm{mmHg})$ & $58 \pm 7$ & $57 \pm 14$ & 0.863 & 1.06 & $0.944-1.072$ \\
\hline Heart rate (beats/min) & $86 \pm 11$ & $85 \pm 15$ & 0.929 & 1.002 & $0.949-1.059$ \\
\hline mPAP $(\mathrm{mmHg})$ & $45 \pm 17$ & $43 \pm 15$ & 0.906 & 1.004 & $0.935-1.079$ \\
\hline PCWP $(\mathrm{mmHg})$ & $21 \pm 7$ & $24 \pm 9$ & 0.478 & 0.969 & $0.887-1.058$ \\
\hline Cardiac index $\left(\mathrm{L} \cdot \mathrm{min}^{-1} \cdot \mathrm{m}^{-2}\right)$ & $2.0 \pm 0.4$ & $2.1 \pm 0.4$ & 0.504 & 0.515 & $0.074-3.606$ \\
\hline $\mathrm{mRAP}(\mathrm{mmHg})$ & $7 \pm 4$ & $8 \pm 5$ & 0.403 & 0.929 & $0.782-1.104$ \\
\hline $\operatorname{RVSWI}\left(\mathrm{g} / \mathrm{m}^{2}\right)$ & $7.7 \pm 3.5$ & $7.7 \pm 3.6$ & 0.951 & 1.007 & $0.817-1.241$ \\
\hline \multicolumn{6}{|l|}{ Echocardiographic parameters } \\
\hline $\operatorname{LVDd}(\mathrm{mm})$ & $78 \pm 12$ & $75 \pm 14$ & 0.445 & 1.022 & $0.966-1.081$ \\
\hline LVEF (\%) & $16 \pm 6$ & $20 \pm 10$ & 0.124 & 0.916 & $0.818-1.025$ \\
\hline AR (grade) & $0.6 \pm 0.7$ & $0.4 \pm 0.7$ & 0.404 & 1.589 & $0.535-4.717$ \\
\hline MR (grade) & $2.4 \pm 1.0$ & $2.3 \pm 1.2$ & 0.849 & 1.067 & $0.548-2.075$ \\
\hline TR (grade) & $1.8 \pm 0.8$ & $1.4 \pm 0.7$ & 0.068 & 2.948 & $0.991-8.752$ \\
\hline Valsalva sinus diameter (mm) & $29 \pm 3$ & $28 \pm 3$ & 0.608 & 1.066 & $0.836-1.358$ \\
\hline $\mathrm{AV}$ ring diameter $(\mathrm{mm})$ & $20 \pm 2$ & $19 \pm 2$ & 0.210 & 1.297 & $0.864-1.947$ \\
\hline \multicolumn{6}{|l|}{ _aboratory parameters } \\
\hline Hemoglobin (g/dl) & $12.5 \pm 2.5$ & $11.2 \pm 2.0$ & 0.121 & 1.313 & $0.930-1.854$ \\
\hline Platelets $\left(\times 10^{3} / \mu \mathrm{l}\right)$ & $19 \pm 7$ & $21 \pm 7$ & 0.454 & 0.957 & $0.854-1.073$ \\
\hline Serum albumin (g/dl) & $3.6 \pm 0.8$ & $3.7 \pm 0.6$ & 0.815 & 0.878 & $0.294-2.624$ \\
\hline Serum sodium (mEq/L) & $136 \pm 3$ & $134 \pm 5$ & 0.239 & 1.126 & $0.924-1.372$ \\
\hline Serum creatinine (mg/dl) & $1.1 \pm 0.5$ & $1.2 \pm 0.6$ & 0.294 & 0.440 & $0.095-2.039$ \\
\hline Serum total bilirubin (mg/dl) & $1.3 \pm 0.6$ & $1.5 \pm 1.2$ & 0.569 & 0.784 & $0.39-1.813$ \\
\hline Plasma BNP (pg/ml) & $839 \pm 673$ & $899 \pm 718$ & 0.812 & 1.000 & $0.999-1.001$ \\
\hline
\end{tabular}

Data given as mean $\pm \mathrm{SD}$ or $\mathrm{n}(\%)$. ${ }^{*} \mathrm{P}<0.05$ (logistic regression). Abbreviations as in Tables 1,2.

been no comprehensive solution for AI, optimal patient/device selection is required in order to prevent $\mathrm{AI}$.

\section{Continuous AV Closure as a Premise for Al}

On early histological examination, continuous AV closure was associated with commissural fusion of native AV, ${ }^{16}$ which resulted in the development of AI. ${ }^{17}$ AI developed consistently only in patients with continuous AV closure (groups $\mathrm{Y}+\mathrm{Z}$; Table 1). Therefore, the first step to prevent AI would be to open native AV during LVAD support.

Opening of native AV is determined by the pressure gradient between the LV and aortic root during the systolic phase. ${ }^{18}$ Elevated pressure in the aortic root due to VAD outflow causes the native AV to remain closed, especially under decreased LV systolic function. Therefore, recovery of LV systolic function is essential for the opening of native AV, especially under already optimized VAD flow with lower rotation speed. After LVAD implantation, those with native AV opening had higher pulse pressure. Such a pressure gradient would be largely dependent on improved LVEF due to the aforementioned LV reverse remodeling under LVAD support.

Considering the early studies, patients with non-ischemic etiology, less fibrosis in myocardium, less dilated LV cavity with shorter HF duration indicating less remodeling, can expect LV reverse remodeling under LVAD support. ${ }^{19-21}$ Preoperative shorter HF duration was consistently a significant predic- 


\begin{tabular}{|c|c|c|c|}
\hline \multirow{3}{*}{ Parameters } & \multicolumn{3}{|c|}{ Continuous AV closure $(n=34)$} \\
\hline & $\mathrm{Al}(+)(\mathrm{n}=11)$ & $\mathrm{Al}(-)(\mathrm{n}=23)$ & P-value \\
\hline & Group Y & Group Z & -value \\
\hline \multicolumn{4}{|l|}{ Postoperative hemodynamics } \\
\hline Heart rate (beats/min) & $82 \pm 10$ & $82 \pm 10$ & 0.934 \\
\hline mPAP $(\mathrm{mmHg})$ & $18 \pm 4$ & $16 \pm 6$ & 0.419 \\
\hline PCWP (mmHg) & $9 \pm 4$ & $8 \pm 4$ & 0.317 \\
\hline Cardiac index $\left(\mathrm{L} \cdot \mathrm{min}^{-1} \cdot \mathrm{m}^{-2}\right)$ & $2.5 \pm 0.7$ & $2.7 \pm 0.6$ & 0.382 \\
\hline mRAP $(\mathrm{mmHg})$ & $8 \pm 5$ & $7 \pm 4$ & 0.714 \\
\hline RVSWI $\left(g / m^{2}\right)$ & $4.0 \pm 1.6$ & $3.9 \pm 2.1$ & 0.924 \\
\hline Pulse pressure (mmHg) & $10 \pm 7$ & $18 \pm 7$ & $0.042^{*}$ \\
\hline $\mathrm{SBP}(\mathrm{mmHg})$ & $87 \pm 6$ & $89 \pm 6$ & 0.389 \\
\hline $\mathrm{DBP}(\mathrm{mmHg})$ & $76 \pm 8$ & $73 \pm 9$ & 0.314 \\
\hline Estimated VAD flow (L/min) & $3.3 \pm 0.6$ & $3.6 \pm 0.9$ & 0.271 \\
\hline Carvedilol at 6 months (mg/day) & $16.4 \pm 14.3$ & $15.2 \pm 13.8$ & 0.824 \\
\hline Enalapril at 6 months (mg/day) & $1.2 \pm 1.5$ & $1.6 \pm 1.6$ & 0.157 \\
\hline \multicolumn{4}{|l|}{ Postoperative echocardiography } \\
\hline \multicolumn{4}{|l|}{ LV and valve } \\
\hline LVDd (mm) & $64 \pm 15$ & $62 \pm 16$ & 0.724 \\
\hline \%change in LVDd (\%) & $-17 \pm 16$ & $-15 \pm 17$ & 0.763 \\
\hline $\operatorname{LVEF}(\%)$ & $15 \pm 6$ & $18 \pm 8$ & 0.335 \\
\hline \%change in LVEF (\%) & $9 \pm 47$ & $-2 \pm 48$ & 0.515 \\
\hline MR (grade) & $0.5 \pm 1.0$ & $0.6 \pm 0.8$ & 0.628 \\
\hline TR (grade) & $1.2 \pm 1.0$ & $1.0 \pm 0.6$ & 0.076 \\
\hline \multicolumn{4}{|l|}{ Aorta and AV } \\
\hline Valsalva sinus diameter $(\mathrm{mm})$ & $32 \pm 3$ & $30 \pm 4$ & 0.096 \\
\hline$\%$ change in Valsalva sinus diameter (\%) & $13 \pm 9$ & $6 \pm 5$ & $0.021^{*}$ \\
\hline $\mathrm{AV}$ ring diameter $(\mathrm{mm})$ & $21 \pm 2$ & $20 \pm 2$ & 0.087 \\
\hline \%change in AV ring diameter (\%) & $9 \pm 8$ & $5 \pm 7$ & $0.048^{*}$ \\
\hline Height of outflow cannula $(\mathrm{cm})$ & $22 \pm 3$ & $23 \pm 3$ & 0.297 \\
\hline
\end{tabular}

Data given as mean $\pm S D$. ${ }^{*}<<0.05$ (unpaired t-test or Mann-Whitney test). Abbreviations as in Tables 1,2.

tor of native AV opening during LVAD support in this study. Shorter HF duration may be associated with less remodeled $\mathrm{LV}$ as well as less preoperative exposure to medical therapy, especially to $\beta$-blockers. ${ }^{22}$ Therefore, those with shorter HF duration may have a greater likelihood of achieving LV reverse remodeling accompanied by native $\mathrm{AV}$ opening due to increased LVEF under hemodynamic unloading on LVAD support and postoperative sufficient medical therapy. Other preoperative predictors of LV reverse remodeling may be found in future studies.

\section{Al During Continuous AV Closure}

In patients with continuous AV closure, AI developed in $33 \%$ (group Y), whereas the remaining 67\% did not have AI (group Z). AI occurred more frequently during axial LVAD support than centrifugal support.

Pulsatility was more reduced during axial LVAD support than during centrifugal support, as noted in other studies. ${ }^{23-25}$ Turbulence, which increases wall shear stress and the retrograde pressure in the aortic root, develops during LVAD support. ${ }^{7,26}$ Degenerative remodeling of aortic root accompanied by thinning of aortic wall emerges, especially under reduced pulsatility due to apoptosis of smooth muscle cells and fragmentation of elastic fibers. ${ }^{27}$ Remodeling of aortic root appears to be associated with higher prevalence of AI as well as degeneration of native AV. ${ }^{14}$ We consistently observed that pa- tients on axial LVAD support had more enlarged aortic root than those with centrifugal support. There were no differences in postoperative LVEF between patients with axial pump and those with centrifugal pump (Table S2). Among those with continuous AV closure, lower pulse pressure might be largely attributable to the use of axial pump.

In other words, pulsatility was relatively preserved during centrifugal LVAD support than axial support. Preserved pulsatility may not only prevent remodeling of aortic root but also reduce diastolic systemic pressure, as shown in the present study, which may reduce diastolic phase retrograde regurgitation through native AV. ${ }^{13}$ An early study in which a lower prevalence of AI was observed during pulsatile LVAD support than CF support, also supported the hypothesis. ${ }^{12}$

\section{Poor Prognosis in Patients With Al}

Toda et al noted worse survival in patients with AI during mainly extracorporeal LVAD support. ${ }^{9}$ AI may contribute to poor survival during extracorporeal VAD support, because AI impairs systemic perfusion and worsens congestion, probably due to the low flow nature of extracorporeal VAD. In contrast, in early studies using mainly CF LVAD, ${ }^{11,28}$ there were no differences in 2-year survival regardless of AI, as observed in the present study. Systemic perfusion may not be decreased even if AI occurs in CF LVAD support. Consistently, we did not observe any differences in hemodynamic parameters be- 


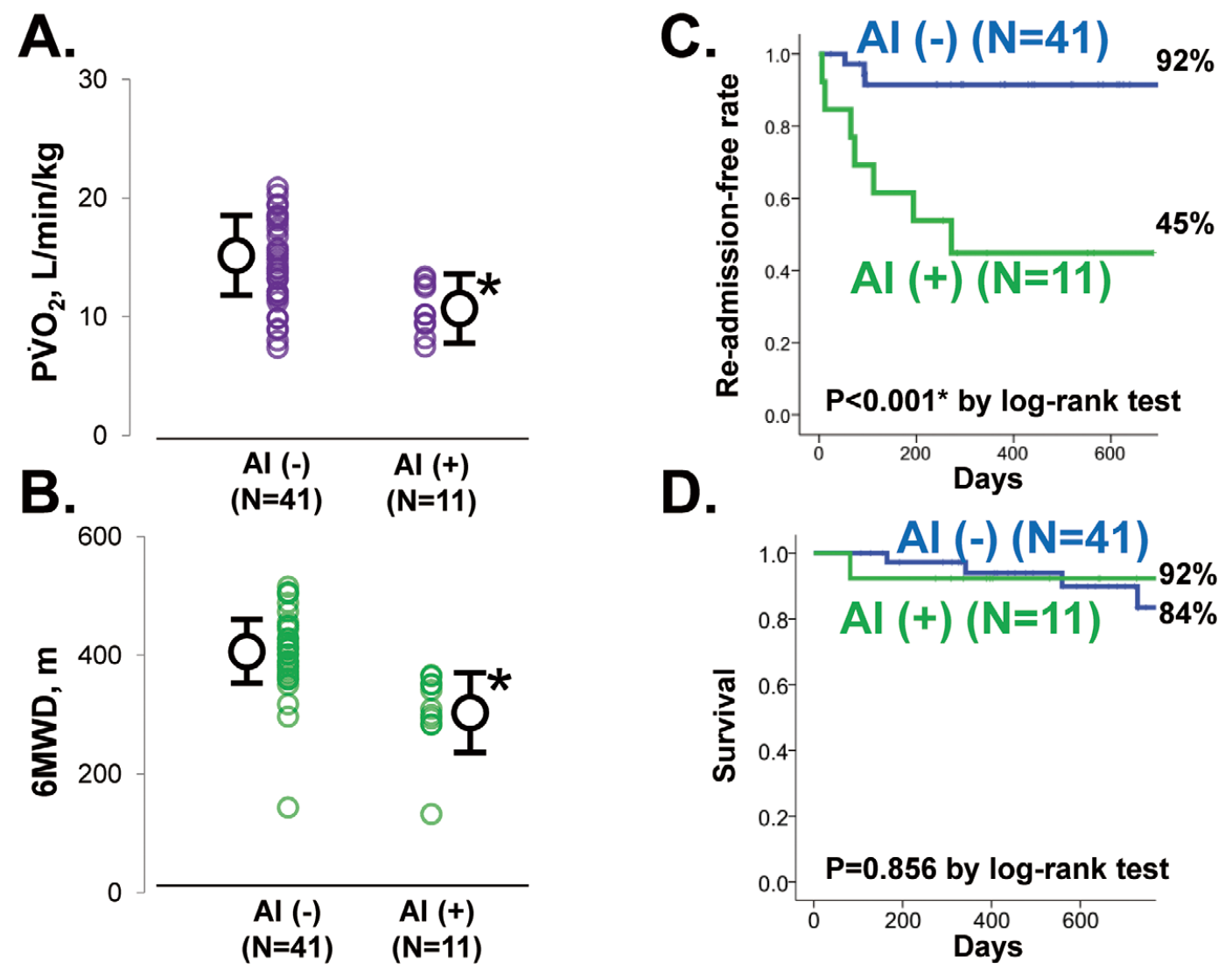

Figure. (A) Peak oxygen consumption ( $\mathrm{PVO}_{2}$ ) and (B) 6-min walk distance (6MWD) at 6 months after left ventricular assist device (LVAD) implantation, and (C) readmission-free rate and (D) survival during 2-year LVAD support among those with/without aortic insufficiency $(\mathrm{Al})$. ${ }^{*} \mathrm{P}<0.05$ (unpaired t-test).

\begin{tabular}{|c|c|c|c|c|}
\hline \multirow[b]{3}{*}{ Aortic valve } & \multicolumn{2}{|c|}{ Centrifugal pump $(n=38)$} & \multicolumn{2}{|c|}{ Axial pump (n=14) } \\
\hline & EVAHEART $(n=24)$ & DuraHeart $(n=14)$ & HeartMate II $(n=11)$ & Jarvik $2000(n=3)$ \\
\hline & & & & \\
\hline AV opening & $11(46)$ & $6(43)$ & $1(9)$ & $0(0)$ \\
\hline $\mathrm{Al}$ & $3(13)$ & $3(21)$ & $5(45)$ & $2(67)$ \\
\hline \multicolumn{5}{|l|}{ Clinical course } \\
\hline $\mathrm{PVO}_{2}\left(\mathrm{ml} \cdot \mathrm{min}^{-1} \cdot \mathrm{kg}^{-1}\right)$ & $14.3 \pm 3.6$ & $13.1 \pm 3.0$ & $13.0 \pm 4.6$ & $9.9 \pm 2.0$ \\
\hline 6MWD $(\mathrm{m})$ & $404 \pm 78$ & $388 \pm 46$ & $361 \pm 104$ & $314 \pm 44$ \\
\hline Re-admission rate (\%) & $4(17)$ & $1(7)$ & $4(36)$ & $2(67)$ \\
\hline
\end{tabular}

Data given as mean $\pm \mathrm{SD}$ or $\mathrm{n}(\%)$. $\mathrm{PVO}_{2}$, peak oxygen consumption; 6MWD, 6-min walk distance. Other abbreviations as in Table 1.

tween patients with and without AI (Table 5).

Patients with AI, however, had decreased exercise capacity compared to those without AI. Although hemodynamics were not different with regard to AI at rest condition, AI may be worsened during exercise with increased afterload. Patients with AI had higher readmission rate due to cardiovascular events such as cerebral thrombosis, ventricular tachyarrhythmia, or congestive HF. Turbulence in ascending aorta due to AI along with continuous AV closure may increase unstable thrombus formation. Increased workload on the LV due to transaortic regurgitation may trigger ventricular tachyarrhythmia.

\section{Study Limitations}

First, data were analyzed in a retrospective manner at a single center, and the sample size was small. The present results should be tested in a prospective manner by randomizing device type in a larger subject group. Second, LVAD were selected by the attending physicians, and selection bias existed. There was no statistical differences, however, in patient background between axial and centrifugal LVAD except for gender and Valsalva sinus diameter. Third, optimization of rotation speed and pre/ postoperative sufficient titration of $\beta$-blocker treatment were carried out in all patients. The present results would not apply 
in situations in which these procedures were not carried out. And fourth, we did not perform AV plasty or replacement to manage developed AI after LVAD implantation. Whether such procedures improve prognosis is a subject for future study.

\section{Conclusions}

Native AV opening during LVAD support is profoundly associated with reversal of LV systolic function, especially in patients with preoperative shorter HF duration. Among those in whom the native AV remains closed, the low pulsatility nature of axial flow pump may facilitate aortic root remodeling and post-LVAD AI development, resulting in poor quality of life.

\section{Acknowledgments}

Grant-in-Aid from Secom Science and Technology Foundation to K.K. K.K. has conflicts of interest as follows. Employment: Daiichi-Sankyo, Otsuka, Terumo, Hitachi-Aloka, CSL Behring, Medix Japan, KCI, Nishimura Kikai, GlaxoSmithKline, Century Medical, Nippon Shinyaku, Edwards, Bayer, Senko Medical Instrument; Research grant: Terumo, Otsuka, ONO, Novartis, Sun Medical; Honoraria: Daiichi-Sankyo, ONO, Otsuka.

\section{References}

1. Imamura T, Kinugawa K, Shiga T, Endo M, Kato N, Inaba T, et al. Preoperative levels of bilirubin or creatinine adjusted by age can predict their reversibility after implantation of left ventricular assist device. Circ J 2013; 77: 96-104.

2. Kirklin JK, Naftel DC, Pagani FD, Kormos RL, Stevenson LW, Blume ED, et al. Sixth INTERMACS annual report: A 10,000-patient database. J Heart Lung Transplant 2014; 33: 555-564.

3. Imamura T, Kinugawa K, Hatano M, Fujino T, Inaba T, Maki H, et al. Low cardiac output stimulates vasopressin release in patients with stage D heart failure. Circ J 2014; 78: 2259-2267.

4. Imamura T, Kinugawa K, Shiga T, Endo M, Kato N, Inaba T, et al. Novel risk scoring system with preoperative objective parameters gives a good prediction of 1-year mortality in patients with a left ventricular assist device. Circ J 2012; 76: 1895-1903.

5. Shiga T, Kinugawa K, Imamura T, Kato N, Endo M, Inaba T, et al. Combination evaluation of preoperative risk indices predicts requirement of biventricular assist device. Circ J 2012; 76: 2785-2791.

6. Deo SV, Sharma V, Cho YH, Shah IK, Park SJ. De novo aortic insufficiency during long-term support on a left ventricular assist device: A systematic review and meta-analysis. ASAIO J 2014; 60: $183-$ 188.

7. Karmonik C, Partovi S, Loebe M, Schmack B, Weymann A, Lumsden $\mathrm{AB}$, et al. Computational fluid dynamics in patients with continuousflow left ventricular assist device support show hemodynamic alterations in the ascending aorta. J Thorac Cardiovasc Surg 2014; 147: $1326-1333$.

8. Jorde UP, Uriel N, Nahumi N, Bejar D, Gonzalez-Costello J, Thomas SS, et al. Prevalence, significance, and management of aortic insufficiency in continuous flow left ventricular assist device recipients. Circ Heart Fail 2014; 7: 310-319.

9. Toda K, Fujita T, Domae K, Shimahara Y, Kobayashi J, Nakatani T. Late aortic insufficiency related to poor prognosis during left ventricular assist device support. Ann Thorac Surg 2011; 92: 929-934.

10. Holtz J, Teuteberg J. Management of aortic insufficiency in the continuous flow left ventricular assist device population. Curr Heart Fail Rep 2014; 11: $103-110$.

11. Aggarwal A, Raghuvir R, Eryazici P, Macaluso G, Sharma P, Blair $\mathrm{C}$, et al. The development of aortic insufficiency in continuous-flow left ventricular assist device-supported patients. Ann Thorac Surg 2013; 95: 493-498.

12. Hatano M, Kinugawa K, Shiga T, Kato N, Endo M, Hisagi M, et al. Less frequent opening of the aortic valve and a continuous flow pump are risk factors for postoperative onset of aortic insufficiency in patients with a left ventricular assist device. Circ J 2011; 75: $1147-1155$.

13. Gregory SD, Stevens MC, Wu E, Fraser JF, Timms D. In vitro evaluation of aortic insufficiency with a rotary left ventricular assist device. Artif Organs 2013; 37: 802-809.

14. Pak SW, Uriel N, Takayama H, Cappleman S, Song R, Colombo PC, et al. Prevalence of de novo aortic insufficiency during long-term support with left ventricular assist devices. J Heart Lung Transplant 2010; 29: $1172-1176$.

15. Slaughter MS, Pagani FD, Rogers JG, Miller LW, Sun B, Russell $\mathrm{SD}$, et al. Clinical management of continuous-flow left ventricular assist devices in advanced heart failure. $J$ Heart Lung Transplant 2010; 29(Suppl): S1-S39.

16. Connelly JH, Abrams J, Klima T, Vaughn WK, Frazier OH. Acquired commissural fusion of aortic valves in patients with left ventricular assist devices. J Heart Lung Transplant 2003; 22: 1291-1295.

17. Martina JR, Schipper ME, de Jonge N, Ramjankhan F, de Weger RA, Lahpor JR, et al. Analysis of aortic valve commissural fusion after support with continuous-flow left ventricular assist device. Interact Cardiovasc Thorac Surg 2013; 17: 616-624.

18. John R, Mantz K, Eckman P, Rose A, May-Newman K. Aortic valve pathophysiology during left ventricular assist device support. J Heart Lung Transplant 2010; 29: 1321-1329.

19. Simon MA, Primack BA, Teuteberg J, Kormos RL, Bermudez C, Toyoda Y, et al. Left ventricular remodeling and myocardial recovery on mechanical circulatory support. J Card Fail 2010; 16: 99-105.

20. Simon MA, Kormos RL, Murali S, Nair P, Heffernan M, Gorcsan J, et al. Myocardial recovery using ventricular assist devices: Prevalence, clinical characteristics, and outcomes. Circulation 2005; 112(Suppl): I32-I36.

21. Matsumiya G, Monta O, Fukushima N, Sawa Y, Funatsu T, Toda K, et al. Who would be a candidate for bridge to recovery during prolonged mechanical left ventricular support in idiopathic dilated cardiomyopathy? J Thorac Cardiovasc Surg 2005; 130: 699-704.

22. Imamura T, Kinugawa K, Hatano M, Fujino T, Muraoka H, Inaba T, et al. Preoperative beta-blocker treatment is a key for deciding left ventricular assist device implantation strategy as a bridge to recovery. J Artif Organs 2014; 17: 23-32.

23. Stanfield JR, Selzman CH. In vitro pulsatility analysis of axial-flow and centrifugal-flow left ventricular assist devices. J Biomech Eng 2013; 135: 34505 .

24. Soucy KG, Koenig SC, Giridharan GA, Sobieski MA, Slaughter MS. Defining pulsatility during continuous-flow ventricular assist device support. J Heart Lung Transplant 2013; 32: 581-587.

25. Moazami N, Fukamachi K, Kobayashi M, Smedira NG, Hoercher $\mathrm{KJ}$, Massiello A, et al. Axial and centrifugal continuous-flow rotary pumps: A translation from pump mechanics to clinical practice. $J$ Heart Lung Transplant 2013; 32: 1-11.

26. Hata H, Fujita T, Ishibashi-Ueda H, Nakatani T, Kobayashi J. Pathological analysis of the aortic valve after long-term left ventricular assist device support. Eur J Cardiothorac Surg 2014; 46: 193-197.

27. Westaby S, Bertoni GB, Clelland C, Nishinaka T, Frazier OH. Circulatory support with attenuated pulse pressure alters human aortic wall morphology. J Thorac Cardiovasc Surg 2007; 133: 575-576.

28. Rajagopal K, Daneshmand MA, Patel CB, Ganapathi AM, Schechter MA, Rogers JG, et al. Natural history and clinical effect of aortic valve regurgitation after left ventricular assist device implantation. $J$ Thorac Cardiovasc Surg 2013; 145: 1373-1379.

\section{Supplementary Files}

Supplementary File 1

Table S1. Preoperative parameters vs. device type

Table S2. Postoperative hemodynamics and echocardiographic parameters vs. device type

Please find supplementary file(s);

http://dx.doi.org/10.1253/circj.CJ-14-0944 